\section{Vol. 30, Issue 8-9, September 2007}

\section{Editorials}

407 Docetaxel Weekly with Metastatic Breast Cancer Twelves, C. (Leeds)

410 Symptom-Oriented Follow-Up of Early Breast Cancer - as Good as Conventional Control and Sparing Resources

Khatcheressian, J., Smith, T.J. (Richmond, VA)

Original Articles

414 Evaluation and Modification of the Tomita Score in 217 Patients with Vertebral Metastases

Ulmar, B.; Reichel, H.; Catalkaya, S.; Naumann, U.; Schmidt, R.; Gerstner, S.; Huch, K. (Ulm)

421 Identification of Consensus Genes and Key Regulatory Elements in 5-Fluorouracil Resistance in Gastric and Colon Cancer

Szöke, D.; Györffy, A. (Budapest); Surowiak, P. (Berlin); Tulassay, Z. (Budapest); Dietel, M. (Berlin); Györffy, B. (Budapest, Berlin)

429 Randomized Controlled Trial of a Structured Training Program in Breast Cancer Patients with Tumor Related Chronic Fatigue

Heim, M.E. (Bad Sooden-Allendorf); Elsner v. d. Malsburg, M (Bad Sooden-Allendorf/Göttingen); Niklas, A. (Göttingen)

436 Improvement of Safety Profile of Docetaxel by Weekly Administration in Patients with Metastatic Breast Cancer

Warm, M. (Köln); Nawroth, F. (Hamburg); Ohlinger, R. (Greifswald); Valter, M. (Köln); Pantke, E. (Aachen); Mallmann, P. (Köln); Harbeck, N.; Kates, R. (München); Thomas, A. (Berlin)

443 Symptom-Oriented Follow-Up of Early Breast Cancer Is not Inferior to Conventional Control. Results of a Prospective Multicentre Study Bornhak, S.; Heidemann, E.; Herschlein, H.J.; Simon, W.; Merkle, E.; Widmaier, G.; Ernst, R.; Greulich, M.; Bittner, R.; Kieninger, G.; Merkle, P.; Strosche, H.; Karg, C.; Wellhaeußer, U.; Aulitzky, W.; Schmidt, B.; Metzger, H.; Hahn, M. (Stuttgart); Stauch, A.; Meisner, C.; Selbmann, H.-K. (Tübingen); Regelmann, C.; Brinkmann, F. (Stuttgart)
Band 30, Heft 8-9, September 2007

Editorials

407 Docetaxel wöchentlich bei metastiertem Brustkrebs Twelves, C. (Leeds)

410 Symptomorientierte Nachsorge nach Mammakarzinom - so gut wie konventionelle Nachsorge und ressourcenschonend Khatcheressian, J., Smith, T.J. (Richmond, VA)

Originalarbeiten

414 Evaluation und Modifikation des Tomita-Scores bei 217 Patienten mit Wirbelsäulenmetastasen Ulmar, B.; Reichel, H.; Catalkaya, S.; Naumann, U.; Schmidt, R.; Gerstner, S.; Huch, K. (Ulm)

421 Identifikation von Konsensus-Genen und Hauptregulatoren der 5-Fluorouracil-Resistenz bei Magen-Darm-Krebs Szöke, D.; Györffy, A. (Budapest); Surowiak, P. (Berlin); Tulassay, Z. (Budapest); Dietel, M. (Berlin); Györffy, B. (Budapest, Berlin)

429 Randomisierte kontrollierte Studie eines Trainingsprogramms bei Brustkrebspatientinnen mit tumorbedingter chronischer Fatigue Heim, M.E. (Bad Sooden-Allendorf); Elsner v. d. Malsburg, M. (Bad Sooden-Allendorf/Göttingen); Niklas, A. (Göttingen)

436 Verbesserung des Nebenwirkungsprofils durch wöchentliche Docetaxelgabe bei Patientinnen mit metastasiertem Mammakarzinom Warm, M. (Köln); Nawroth, F. (Hamburg); Ohlinger, R. (Greifswald); Valter, M. (Köln); Pantke, E. (Aachen); Mallmann, P. (Köln); Harbeck, N.; Kates, R. (München); Thomas, A. (Berlin)

443 Symptomorientierte Nachsorge nach Mammakarzinom ist der konventionellen Nachsorge nicht unterlegen. Ergebnisse einer prospektiven multizentrischen Studie Bornhak, S.; Heidemann, E.; Herschlein, H.J.; Simon, W.; Merkle, E.; Widmaier, G.; Ernst, R.; Greulich, M.; Bittner, R.; Kieninger, G.; Merkle, P.; Strosche, H.; Karg, C.; Wellhaeußer, U.; Aulitzky, W.; Schmidt, B.; Metzger, H.; Hahn, M. (Stuttgart); Stauch, A.; Meisner, C.; Selbmann, H.-K. (Tübingen); Regelmann, C.; Brinkmann, F. (Stuttgart)

\begin{tabular}{|c|c|}
\hline KARGER & (c) 2007 S. Karger GmbH, Freiburg \\
\hline $\begin{array}{l}\text { Fax +49761 } 4520714 \\
\text { E-mail Information@Karger.de } \\
\text { www.karger.com }\end{array}$ & $\begin{array}{l}\text { Artikel (Volltext) und Inhaltsverzeichnisse } \\
\text { sowie das vorläufige Inhaltsverzeichnis des nächsten Heftes: } \\
\text { www.karger.com/onk_bk.htm }\end{array}$ \\
\hline
\end{tabular}




\section{Vol. 30, Issue 8-9, September 2007}

Clinical Cases

450 Renal Cell Cancer Presented with Leptomeningeal Carcinomatosis Effectively Treated with Sorafenib Ranze, O.; Hofmann, E.; Distelrath, A.; Hoeffkes, H.-G. (Fulda)

452 Paravasation with Cyclophosphamide a Case Report of Tissue Necrosis in a Patient with Primary Breast Cancer Jueckstock, J.K.; Mylonas, I.; Strobl, B.; Willgeroth, F.; Sommer, H.L.; Friese, K. (München)

455 Syndrome of Inappropriate Antidiuretic Hormone Secretion (SIADH) Induced by Vinorelbine Treatment of Metastatic Breast Cancer

Canzler, U.; Schmidt-Göhrich, U.K.; Bergmann, S.; Hänseroth, K.; Gatzweiler, A.; Distler, W. (Dresden)

\section{Letter to the Editors}

457 Adenocarcinoma of the Appendix and a Meckel's Diverticulum in a Case of Acute Appendicitis Lochman, P.; Cáp, R.; Kohout, A. (Hradec Králové)

\section{Book Reviews}

461 PharmaNews / PharmaTicker

463 PharmaForum

466 Meetings and Conferences

406 Imprint

Forthcoming papers are listed on page 468 .

\section{Band 30, Heft 8-9, September 2007}

Kasuistiken

450 Erfolgreicher Einsatz von Sorafenib zur Behandlung eines Nierenzellkarzinom mit Meningeosis carcinomatosa Ranze, O.; Hofmann, E.; Distelrath, A.; Hoeffkes, H.-G. (Fulda)

452 Paravasat nach Cyclophosphamidapplikation Ein Fallbericht einer Gewebsnekrose Jueckstock, J.K.; Mylonas, I.; Strobl, B.; Willgeroth, F.; Sommer, H.L.; Friese, K. (München)

455 Syndrom der inadäquaten ADH-Sekretion (SIADH) unter Therapie mit Vinorelbin bei metastasiertem Mammakarzinom Canzler, U.; Schmidt-Göhrich, U.K.; Bergmann, S.; Hänseroth, K.; Gatzweiler, A.; Distler, W. (Dresden)

Brief an die Herausgeber

457 Adenokarzinom der Appendix und Meckeldivertikel bei einem Patienten mit akuter Appendizitis Lochman, P.; Cáp, R.; Kohout, A. (Hradec Králové)

459 Buchbesprechungen

461 PharmaNews / PharmaTicker

463 PharmaForum

466 Tagungen und Kongresse

406 Impressum

Einen Ausblick auf den Inhalt der kommenden Hefte finden Sie auf Seite 468.

\section{KARGER}

Fax +497614520714

E-mail Information@Karger.de

www.karger.com
() 2007 S. Karger GmbH, Freiburg

Artikel (Volltext) und Inhaltsverzeichnisse

sowie das vorläufige Inhaltsverzeichnis des nächsten Heftes: www.karger.com/onk_bk.htm 